\title{
La prevención y los presupuestos personales en la prestación de Servicios Sociales: dos áreas potenciales de generación de eficiencia. La experiencia del Reino Unido
}

\author{
Prevention and personal budgets in the provision of Social Care Public \\ Services: Two potential areas in the achievement of efficiency. \\ The United Kingdom's experience
}

\author{
Carolina Pontones Rosa \\ Universidad de Castilla-La Mancha \\ carolina.pontones@uclm.es
}

Recibido: 20/06/2012

Revisado: 02/08/2012

Aceptado: 12/03/2013

Disponible on line: 20/12/2013

\section{Resumen}

El Reino Unido se ha constituido como uno de los países clave en el impulso de los principios inspiradores de la Nueva Gestión Pública y la Gobernanza de los Servicios Sociales fomentando los principios de eficiencia, eficacia y transparencia. A su vez, el escenario económico y social actual hace que, tanto en el Reino Unido como en España y demás países europeos, más que nunca, sea necesario potenciar técnicas y políticas de prestación de Servicios Sociales que ayuden a ampliar la cobertura de los mismos con una mayor calidad y un menor coste en la prestación.

Ante una tarea tan complicada el principal objetivo de este artículo se centra en describir dos de estas técnicas utilizadas en Reino Unido y contempladas como fuentes potenciales de generación de eficiencia. Dichas técnicas se refieren, por un lado, a la inversión en políticas de prevención destinadas a desarrollar servicios de corta duración consistentes en ayuda a domicilio especializada e intensiva, cuya finalidad es la recuperación de capacidades. Y por otro lado, se plantea el Sistema de Presupuestos Personales como una nueva tendencia en la que los individuos tienen capacidad de elección y control, participando de forma activa en la configuración de los servicios que precisan.

Palabras clave: Servicios Sociales, Nueva Gestión Pública, Reino Unido, Recapacitación, Presupuesto Personal.

\begin{abstract}
The United Kingdom has become one of the leading countries in the thrust of the New Public Management and Governance principles, promoting value for money and transparency in the provision of social care public services. In turn, the current social and economic scenery poses, more than ever, the need for European countries, particularly for Spain, to improve social services' delivery in order to cover the increasing demand at a lower cost and maintaining the levels of satisfaction.

Against such a complicated goal, the aim of this paper is to depict two of the main initiatives undertaken by Local Government in the United Kingdom, which are currently regarded as sources of efficiency. These techniques are referred to, on the one hand, the investment in prevention policies to develop short term interventions, mainly specialized and intensive home care attention - reablemet - and, on the other, the implementation of personal budgets within the Self-directed Support System, as a new trend both to enhance choice and control for the users and to raise their role in shaping their care packages.
\end{abstract}

Keywords: Social care services, New Public Management, United Kingdom, Reablement, Personal Budget.

Referencia normalizada: Pontones Rosa, C. (2013): «La prevención y los presupuestos personales en la prestación de Servicios Sociales: dos áreas potenciales de generación de eficiencia. La experiencia del Reino Unido». Cuadernos de Trabajo Social, 26(2): 385-395.

Sumario: Introducción. 1. La prevención en los Servicios Sociales como herramienta estratégica para la reducción de costes en Reino Unido. 2. El Sistema Self-directed Support o de Apoyo Auto-dirigido. 3. Conclusiones. 4. Referencias bibliográficas. 


\section{Introducción}

El escenario de crisis económica y social actual que vivimos marca las líneas de austeridad en las que viene desarrollándose la gestión pública en general y de los Servicios Sociales en particular. El objetivo de déficit presupuestario ha sometido a una gran rigidez la prestación de dichos servicios, generando una dinámica de actuación que difícilmente da respiro a la obtención de unos objetivos de calidad razonables. Por todo ello, se hace necesaria, más que nunca, la utilización de técnicas de gestión encaminadas a la disminución de costes y, por lotanto, de una mayor eficiencia, sin perder de vista la necesidad de ampliar al máximo su cobertura a la ciudadanía con la mayor eficacia posible.

Los paradigmas de la Nueva Gestión Pública y de la Gobernanza se conciben como las directrices que deben regir la gestión de los Servicios Sociales teniendo en cuenta la creciente complejidad social y la concurrencia de una pluralidad de actores que deben ser incluidos en la gestión de las políticas públicas (García, 2007). En este sentido, tanto España como otros países de nuestro entorno han experimentado problemas de falta de eficiencia y agilidad en la prestación de los Servicios Sociales, a los que se añaden las previsiones socio-demográficas que indican un crecimiento continuado de la demanda (Hughes, 2003). No es de extrañar que los estudios relativos a los principios de eficacia, eficiencia y transparencia se encuentren en continua renovación, habiéndose iniciado su desarrollo empírico en el Reino Unido (Jennergrem, 1981; Hogwood y Peters, 1985; Osborne, 2000).

Los trabajos de Antonnen y Sipila (1996) y Casado y Fantova (2007), que realizan un análisis de las principales tendencias internacionales en Servicios Sociales, destacan al Reino Unido como país emprendedor de interesantes reformas en la concepción, organización y gestión de los Servicios Sociales, con el objeto de elevar la economía, eficacia y eficiencia de su prestación. En concreto, destacan iniciativas como la separación commissioner-provider, la proliferación del mercado privado de Servicios Sociales, la creación de consorcios o partnerships locales para la prestación de servicios, o de novedosos modelos de atención tendentes a la personalización y la prevención ${ }^{1}$.

Paralelamente, en las últimas décadas el Reino Unido ha fomentado la implantación de una cultura de mejora de la gestión de la Administración Local. En este proceso, destaca la promulgación de regulaciones sobre el sector público local que han abierto la puerta a la comparabilidad, el aprendizaje y la mejora del denominado value for money ${ }^{2}$, tomando como base el establecimiento de requisitos obligatorios de emisión de información, que han sido reforzados por sucesivos regímenes de auditoría operativa externa. Se trata de un modelo de medición de resultados y control que se halla en constante evolución y al que se le reconocen importantes avances y ventajas, pero que también ha sido objeto de críticas. Dado su interés, el Reino Unido ha sido seleccionado como país objeto de estudio en relación con los Servicios Sociales y la gestión pública.

El objetivo de este artículo es poner de manifiesto la contribución de dos de las técnicas más utilizadas en la prestación de los Servicios Sociales en dicho país, de cara a la consecución de los principios de eficiencia y eficacia anteriormente mencionados. Estas técnicas se sustentan en dos pilares básicos: la prevención, como herramienta estratégica de mejora del bienestar y de reducción de costes, y la personalización a través del uso de presupuestos personales. Ambos han sido identificados por la Audit Commission $(2011)^{3}$ como áreas potencialmente generadoras de eficiencia.

En el año 2009 el Gobierno central de Inglaterra (HM Government) hizo público el documento Shaping the Future of Care Together y se celebró la mayor consulta pública en materia de protección social, el Big Care Debate, donde participaron 68.000 personas, aproximadamente. La opinión ciudadana puso de

\footnotetext{
${ }^{1}$ Puede consultarse al respecto, Martínez (2000) y Flynn (2000).

${ }^{2}$ El término anglosajón value for money, aplicado al ámbito público, hace referencia a la gestión eficaz, económica y eficiente de los recursos.

${ }^{3}$ La Audit Commission es el organismo encargado de la auditoría externa del sector público local en Reino Unido.
} 
manifiesto en ella la necesidad de llevar a cabo reformas integrales para hacer frente a los retos demográficos, tecnológicos y económicos que se le planteaban.

En el Reino Unido, el proceso de transformación emprendido en los Servicios Sociales para adultos ${ }^{4}$ ha avanzado en este camino y las líneas actuales giran en torno a la creación de un Servicio Nacional de Cuidado (HM Government, 2010) basado en seis pilares fundamentales:

- Servicios de prevención y bienestar para promover la independencia.

- Criterios de elegibilidad ${ }^{5}$ uniformes a nivel nacional y recogido en la legislación.

- Valoración única y conjunta.

- Información y consejo sobre recursos.

- Apoyo y cuidados personalizados a través del presupuesto personal.

- Financiación justa del sistema, basada en una responsabilidad compartida mediante un régimen contributivo.

La reforma se construye sobre la base de numerosos proyectos y publicaciones elaborados por el gobierno central, entre los que destaca la puesta en marcha de la agenda Putting People First (Department of Health, 2007), de aplicación en Inglaterra en el campo de los Servicios Sociales para adultos ${ }^{6}$. Esta iniciativa promueve el cambio integral en la forma en que la comunidad, las organizaciones y los individuos prestan apoyo a aquéllos que lo necesitan o pueden necesitarlo. El proyecto gira en torno a cuatro áreas que describimos a continuación:

- Fomentar el desarrollo de los servicios públicos universales.
- Énfasis en la prevención y la intervención temprana.

- Elevar la capacidad de elección y control a través de la personalización.

- Desarrollo del capital social.

De todas ellas haremos referencia, a continuación, a la segunda y la tercera.

\section{La prevención en los Servicios Sociales como herramienta estratégica para la re- ducción de costes}

Los gobiernos locales han de considerar las necesidades globales de la población y diseñar estrategias para reducir el número de personas que acceden al Sistema de Servicios Sociales por primera vez. Los servicios preventivos, en general, suelen conllevar cierta inversión inicial para propiciar cambios que, con el tiempo, reduzcan la necesidad de intervención, con la subsiguiente disminución de costes. Por este motivo, han sido considerados como un mecanismo de constricción del gasto público. No obstante, como señala Prowle (2010), estos servicios sólo generarán value for money si su diseño, implementación y evaluación están orientados a la economía, la eficiencia y la efectividad, entendida como eficacia e impacto.

Un ejemplo de actuación preventiva es el servicio de recapacitación o reablement, que es ofertado por un número cada vez mayor de gobiernos locales. En muchos de ellos, los departamentos de Servicios Sociales para adultos están realizando una evolución desde los servicios de ayuda a domicilio tradicionales hacia estos servicios preventivos. El reablement engloba un conjunto de servicios de corta duración consistentes en ayuda a domicilio especializada e intensiva, cuya finalidad es la

${ }^{4}$ En la mayor parte del Reino Unido, los Servicios Sociales se encuentran divididos en Servicios Sociales para adultos (Adult Social Care Services) y Servicios Sociales para niños (Children Social Care Services). Esta división afecta a su financiación, organización y prestación.

${ }^{5}$ Los Servicios Sociales ofertados por la Administración Local pueden ser financiados con fondos públicos, de acuerdo con el criterio de elegibilidad. Este criterio puede definirse como el conjunto de necesidades que son susceptibles de satisfacción por parte de los gobiernos locales, considerando sus disponibilidades presupuestarias.

${ }^{6}$ Con el objeto de dar cobertura económica a la agenda Putting People First, se concedió financiación específica a través de la «Transferencia para la Reforma de los Servicios Sociales» —Social Care Reform Grant - en el período 2008-2011. Asimismo, para orientar en el proceso de transformación, identificar y desarrollar formas más eficientes de prestar los servicios, el Ministerio de Sanidad puso en marcha equipos de apoyo denominados Care Services Efficiency Delivery Teams que han estado operativos hasta marzo de 2011. 
recuperación de capacidades. Se trata de una nueva filosofía o enfoque que tiene por lema «ayudar a los usuarios a que hagan las cosas por sí mismos».

Una definición de reablement generalmente aceptada los entiende como «servicios para personas de salud física y mental reducida, destinados a ayudarles a mejorar sus condiciones de vida a través del aprendizaje o re-aprendizaje de las habilidades necesarias para la vida cotidiana» (Kent, Payne, Stewart y Unelll, 2000). Estos servicios atienden necesidades que recaen en un área común a los servicios sociales y sanitarios. En la medida en que las personas sean capaces de vivir de forma independiente, su demanda de ambos tipos de servicios se reducirá. Por este motivo, en algunas áreas locales los servicios de recapacitación son financiados y gestionados conjuntamente por los gobiernos locales y el Servicio Nacional de Salud. Según datos del Department of Health (2010) aproximadamente un cuarto de los servicios cuentan con parte de financiación procedente del Sistema Sanitario, mientras que el resto son asumidos en su totalidad por los gobiernos locales.

Una investigación llevada a cabo por la $S o$ cial Policy Research Unit, University of Kent (2010), examinó los efectos inmediatos y a largo plazo del servicio de reablement en ayuda a domicilio, su coste-efectividad, su contenido y organización. Durante un año, se realizaron comparaciones entre un grupo de usuarios que habían recibido previamente reablement (grupo 1) y otro grupo de usuarios que, durante el período analizado, se encontraban recibiendo servicios convencionales de ayuda a domicilio de forma continuada (grupo 2 ). Algunas de las conclusiones obtenidas fueron las siguientes:

- La recepción de servicios de recapacitación se asociaba a un descenso significativo en la demanda posterior de Servicios Sociales.

- El coste de los Servicios Sociales recibidos por el grupo 1 durante el período analizado fue un 60 por ciento menor que el coste del grupo 2. Sin embargo, la reducción en costes era compensada casi en su totalidad por la inversión inicial en la intervención de recapacitación.

- Al considerar conjuntamente los costes de servicios sanitarios y servicios sociales
- tanto los de servicios convencionales como los de reablement - recibidos durante el período de estudio, no se encontraron diferencias estadísticamente significativas entre los dos grupos.

- La recapacitación presentó efectos positivos en la calidad de vida del grupo 1, tanto respecto a necesidades atendidas por los servicios sociales como por los sanitarios, en los diez meses posteriores a su recepción, en comparación con el grupo 2 .

Junto a estas conclusiones, el Care Services Efficiency Delivery Team (CSED) realizó un estudio sobre servicios de reablement, en el cual se concluyó que hasta un 50 por ciento de personas mayores receptoras de este tipo de prestaciones durante un breve período de tiempo no requirió más intervenciones al finalizar su tratamiento (Department of $\mathrm{He}$ alth, 2009).

Para que el servicio de reablement sea efectivo, es necesario poner en marcha planes de formación y entrenamiento del personal adecuados, establecer mecanismos de supervisión continua, definir con claridad los resultados a alcanzar por los usuarios, dotar a los objetivos de flexibilidad para adaptarse a eventuales cambios en las necesidades $y$, disponer de una dotación de equipamientos adecuados. La transferencia rápida a los servicios de ayuda a domicilio para aquéllos que todavía lo necesiten, una vez finalizado el servicio de recapacitación, es esencial para mantener la capacidad de estos servicios preventivos.

En el documento Prioritising need in the context of Putting People First: A whole system approach to eligibility for social care, el Department of Health (2010a, p.18), recomienda a los gobiernos locales que, antes de aplicar el criterio de elegibilidad a un nuevo caso, se planteen si sería beneficioso para incrementar la autonomía del individuo recibir el servicio de recapacitación o de cuidado intermedio para, posteriormente y una vez concluido el servicio, llevar a cabo la valoración de sus necesidades a largo plazo y determinar si son elegibles para acceder a la oferta pública.

Dentro de los servicios preventivos, los gobiernos locales han de considerar también la posibilidad de proporcionar apoyo al cuidador de la persona que presenta el problema, como 
vía para reducir la necesidad de intervenciones más intensivas.

Asimismo, los servicios denominados extracare housing - alojamiento con apoyo- prestados en cooperación con los Servicios de Vivienda, también constituyen servicios de prevención. Se trata de alojamientos creados ad hoc en los que se encuentran disponibles diferentes grados de apoyo y donde existe acceso a algunos servicios e instalaciones compartidos. Las viviendas tienen que ser adquiridas o alquiladas por los usuarios, limitándose la actuación de los gobiernos locales a la mediación con las entidades o agencias que prestan los cuidados a domicilio y con el arrendador de la vivienda, en su caso.

La obtención de eficiencia a través de la prevención está asociada a la capacidad de «situar a la persona adecuada en la parte oportuna del Sistema y en el momento óptimo»». De esta forma no sólo se generará eficiencia, sino que se obtendrán mejores resultados para los usuarios. En conclusión, las acciones preventivas bien diseñadas son más interesantes en términos de coste-beneficio, sobre todo si tenemos en cuenta el aumento del bienestar que generan (Department of Health, 2009).

\section{El sistema de presupuestos personales (Self-directed Support) o de apoyo auto-di- rigido}

El Self-directed Support (SDS) o Apoyo Autodirigido es una nueva filosofía que propugna el cambio hacia un Sistema de Servicios Sociales personalizado, en el que los individuos tengan capacidad de elección y control, desarrollando sus propias soluciones y participando de forma activa en la configuración de los servicios que precisan. Esta nueva concepción es una parte del proyecto Putting People First (Department of Health, 2007) en el contexto de la reforma global del Sistema Público de Servicios Sociales.

Entre los objetivos del sistema Self-directed Support se encuentran los siguientes (Hampshire County Council, 2009):
- Ofrecer información de calidad a los usuarios sobre la oferta de recursos y de servicios disponibles.

- Permitir a las personas un mayor control de los recursos de que disponen.

- Proporcionar planes de apoyo en los que se asesore y oriente a los individuos sobre cómo emplear los recursos disponibles para dar respuesta a sus necesidades.

- Mantener la oferta tradicional de Servicios Sociales u otras formas de apoyo comunitario en el caso de que se opte por dicha posibilidad.

- Permitir a los usuarios decidir sobre cómo afrontar sus problemáticas y elegir los servicios que necesitan para ello.

Para su consecución, el Self-directed Support se basa en una nueva fórmula de asignación de recursos denominada genéricamente «presupuesto personal», que ha de ofrecerse a todos los usuarios - elegibles - de forma obligatoria $^{7}$. A través de un sistema de asignación de recursos que permite traducir en cantidades monetarias las valoraciones de las problemáticas emitidas por los profesionales de los Servicios Sociales, se procede al cálculo del importe concedido para financiar sus servicios. Esto se pone en conocimiento de los usuarios - $-\mathrm{y}$ sus tutores u orientadores, en su caso- $y$, desde ese momento, el individuo será beneficiario de un presupuesto personal ${ }^{8}$. Aunque esta cantidad debe ser suficiente para permitir al usuario adquirir los servicios que requieren sus necesidades, en muchos casos el receptor contribuirá en parte al coste de su cuidado9.

Considerando la información anterior, el individuo, con la ayuda de los profesionales, procede a elaborar un plan para la resolución de sus problemáticas a partir de la definición de objetivos concretos a alcanzar. El plan debe ser acordado entre todas las partes.

La siguiente fase implica la elección por el usuario de cómo organizará el dinero asignado

\footnotetext{
${ }^{7}$ En el caso de los Servicios Sociales para niños, no se ha anunciado su implantación obligatoria a nivel nacional, aunque en algunas corporaciones se están desarrollando a modo de prueba.

${ }^{8}$ Los familiares y amigos que se ocupan de forma voluntaria del cuidado diario de enfermos o usuarios de Servicios Sociales también pueden ser receptores de un presupuesto personal cuando, debido a tal labor, hayan desarrollado problemáticas y necesidades consideradas elegibles.

${ }^{9}$ Los gobiernos locales deberán aplicar la guía denominada Fairer Contributions Guidance: Calculating an Individual's Contribution to their Personal Budget, (Department of Health, 2010c)
} 
en su presupuesto personal, pudiendo acogerse a tres modalidades (Nottinghamshire County Council, 2010a):

- Pago directo: supone la entrega directa de los fondos al usuario, en lugar de prestarle el servicio. El dinero podrá ser gestionado por él mismo o por otra persona o entidad en su nombre.

- Presupuesto personal gestionado: el departamento de Servicios Sociales puede gestionar el presupuesto personal por el individuo, comisionando los servicios oportunos.

- Presupuesto personal mixto: una parte de los fondos se entrega como pago directo y el resto es gestionado por el gobierno local.

Además, dentro de la casuística que puede surgir en el uso de los presupuestos personales, se contempla la posibilidad de que el usuario, $o$ alguien que lo represente, entregue el dinero a una entidad prestadora de Servicios Sociales para que lo gestione en su nombre y de acuerdo con sus indicaciones. Esta modalidad se denomina Individual Service Fund.

También existe la figura del presupuesto individual que, conceptualmente, es similar a la del presupuesto personal. Sin embargo, mientras que este último sólo recoge fondos procedentes del Sistema Público de Servicios Sociales para Adultos, el presupuesto individual engloba una variada gama de fondos de distinta procedencia ${ }^{10}$. Esta modalidad se creó para facilitar a determinados colectivos, como los discapacitados, la obtención de ayudas por diferentes organismos. Su desarrollo requiere que dichos organismos acuerden procedimientos comunes de valoración de necesidades, aspecto en el que se está trabajando. Los presupuestos individuales sólo se están aplicando, a modo de prueba, en adultos, niños discapacitados y sus familias.

Una vez determinada la forma en la que el usuario va a recibir y gestionar su presupuesto personal, contará con el asesoramiento del departamento de Servicios Sociales para orientarle sobre los servicios que puede contratar para la solución de sus problemáticas. Se informará al usuario sobre la oferta de Servicios Sociales disponible para atender su problema, teniendo en cuenta que la elección realizada dependerá en gran medida de la oferta que se le oferte al ciudadano, la información y el asesoramiento prestado para apoyar sus decisiones (Henwood, 2008). El requisito de imparcialidad debe respetarse en cualquier caso, tanto si el apoyo necesario es ofertado por el propio Gobierno Local in-house como si no, ofreciendo un diagnóstico sin limitaciones ni restricciones de cualquier índole ${ }^{11}$. Si se trata del dinero del propio usuario, la tarea de los profesionales se limitará a valorar e informar de la oferta local de servicios y de la idoneidad de la misma para el caso.

Desde una perspectiva general, según el documento Prioritising need in the context of Putting People First: A whole system approach to eligibility for social care (Departamento de Sanidad, 2010, p. 41), la implementación de los presupuestos personales requiere el diseño de un sistema de asignación de recursos ${ }^{12}$ que permita determinar, a partir de las necesidades del individuo, la cantidad a asignar en forma de presupuesto personal. Un factor clave de éxito en el desarrollo de este sistema es el logro de la transparencia en el proceso de cálculo y la forma como se vinculan los fondos concedidos a los resultados esperados. A través del mismo debería proporcionarse un importe in-

${ }^{10}$ Fundamentalmente procedentes de las prestaciones sociales denominadas Support People, que es una ayuda a la vivienda en alquiler y Disabled Facilities Grant, destinada a realizar adaptaciones en el hogar para discapacitados.

${ }^{11}$ Existe una guía dirigida a las autoridades locales para que estas funciones se lleven a cabo de forma segura, permitiendo la prestación un apoyo adecuado a los usuarios en su toma de decisiones: Independence, choice and risk: a guide to best practice in supported decision making. Department of Health (2007a)

${ }^{12}$ La Asociación de Directores de Servicios Sociales para Adultos (ADASS), que representa a los directores de Servicios Sociales para Adultos de Inglaterra, ha estado trabajando con el Programa In Control y dieciocho gobiernos locales para desarrollar un sistema común de asignación de recursos, de adopción voluntaria para los gobiernos locales (ADASS, 2009). No obstante, es poco probable la implantación de un único sistema en toda Inglaterra, dadas las diferencias existentes en cuanto a circunstancias locales. En este sentido, el Departamento de Sanidad se ha comprometido a colaborar con el Gobierno local para la puesta en común de buenas prácticas en este campo a nivel nacional. 
dicativo del coste de los servicios requeridos por el individuo, mostrando suficiente flexibilidad ante las circunstancias de cada persona. Por otro lado, en la configuración del sistema de asignación de recursos, los departamentos de Servicios Sociales deberían emprender un análisis crítico sobre cómo desarrollan su función de commissioning ${ }^{13}$, planteándose si ésta se lleva a cabo en condiciones de economía y eficiencia y en qué medida los costes de los Servicios Sociales contratados han podido verse incrementados por una gestión inadecuada.

Por otro lado, una vez seleccionada la modalidad de uso del presupuesto personal y, transcurrido un tiempo suficiente, el usuario deberá demostrar el grado de consecución de los objetivos marcados, así como que ha utilizado el dinero de la forma prevista. Esto se realiza mediante revisiones periódicas, en las que pueden introducirse cambios en el plan de apoyo si es necesario ${ }^{14}$. La forma como ha de llevarse a cabo la evaluación del empleo y resultados de los presupuestos personales por parte de los gobiernos locales no está regulada y ello abre el debate sobre la delegación de responsabilidad por parte de los poderes públicos, especialmente bajo la opción de pagos directos, al no asegurar la efectiva prestación y recepción de Servicios Sociales por parte de los ciudadanos. No existe certeza sobre si los usuarios serán capaces de diseñar servicios de calidad que respondan a sus problemáticas de manera eficiente, ni si decidirán emplear los fondos para los fines para los que fueron concedidos.

A pesar de que este sistema trata de fomentar la auto-responsabilidad, algunas personas pueden necesitar una ayuda extra para gestionar su apoyo, ya sea a través de abogados o defensores. En estos casos, es habitual que el departamento de Servicios Sociales contrate el servicio con una entidad privada o con los propios Servicios Sociales públicos, si fuera conveniente, actuando en nombre del usuario y gestionando directamente su presupuesto personal. De este modo, se asegura que el individuo utiliza el dinero en el fin adecuado, ya sea porque se trata de una persona en la que no se puede confiar $o$, en especial, cuando se trate de personas incapacitadas para decidir por sí mismas y se estime riesgo de que el dinero pudiera ser gestionado por familiares en su propio interés.

Para los gobiernos locales y el central, los pagos directos constituyen la opción preferente en el uso de presupuestos personales en la actualidad (Department of Health, 2010b) y se espera que mejore su recepción en los próximos años, al considerarse la opción más favorable en términos de coste y eficacia.

Aunque el sistema Self-directed Support fue introducido en 2007, los pagos directos son de oferta obligatoria para la mayoría de grupos de usuarios desde $2003^{15}$ y fueron introducidos en el ámbito de los Servicios Sociales en 1996. Su principal ventaja es que aumentan la capacidad de elección de los individuos. Su fórmula es más flexible que el presupuesto personal gestionado, dado que el Departamento de Servicios Sociales sólo contrata servicios con un rango limitado de entidades prestadoras.

Un rasgo distintivo de los pagos directos es que confieren responsabilidades a sus receptores a la hora de contratar personas - denominadas asistentes personales_- ${ }^{16}$ o servicios para

${ }^{13}$ La función de commissioning implica que los gobiernos locales evalúen sistemáticamente la conveniencia de prestar directamente los Servicios Sociales o encargarlos a otras entidades. Su misión es promover la proliferación de servicios de alta calidad que, por un lado, eleven las alternativas de elección para el individuo y, por otro, generen ganancias de eficiencia a través de la competencia.

${ }^{14}$ En este sentido, las autoridades locales están recibiendo apoyo desde el Departamento de Sanidad a través del DH's Deputy Regional Director for Social Care and Partnerships - para desarrollar sistemas de control de ejecución que midan los beneficios obtenidos por los usuarios y la comunidad derivados de la personalización del sistema y capaces de aportar datos fiables que puedan ser usados por los procesos de evaluación, proporcionando información útil para la estrategia de commissioning (Department of Health, Local Authorities Circular, 2009a, 1).

${ }^{15}$ A partir de las Regulaciones de 2003 denominadas The Community Care, Services for Carers and Children's Services (Direct Payments) (England) Regulations 2003. Sin embargo, su aplicación no se contemplaba para los casos de servicios residenciales de larga duración ni de servicios prestados por el propio departamento de Servicios Sociales.

${ }^{16}$ La persona contratada no puede residir en la misma vivienda que el usuario. 
su apoyo. De esa forma, el usuario asume todas las cargas propias de un empleador, como por ejemplo, el pago de nóminas, el cumplimiento de requisitos legales tales como la garantía del salario mínimo, así como la tramitación del contrato de trabajo. Algunas de estas tareas pueden ser delegadas a terceros y muchos gobiernos locales tienen establecidos acuerdos con organizaciones que ayudan a los usuarios a afrontar dichas responsabilidades. El resto de modalidades de presupuesto personal eximen de las responsabilidades derivadas de estos últimos, en cuanto a contratación se refiere.

La aceptación de los pagos directos se ha elevado significativamente en los últimos años, aunque todavía permanece baja en proporción al total de personas receptoras de servicios. La baja respuesta ha sido explicada de dos formas (Mithran, 2009):

- La baja predisposición para promover los pagos directos y el establecimiento de obstáculos burocráticos por parte de los gobiernos locales, unido al reducido interés de una parte de los usuarios a optar por esta vía debido a las cargas - reales o percibidas - que conlleva.

- La cautela por el riesgo derivado de la contratación de asistentes personales no idóneos. A pesar de que casi el 50 por ciento de los usuarios de pagos directos no consulta los registros de antecedentes penales a la hora de contratar asistentes personales y que el 46 por ciento no suele buscar ningún tipo de referencia, en la actualidad no se prevé el establecimiento de normativas que obliguen a los usuarios a someter a controles a aquéllos a los que contratan para su cuidado (Lombard, 2008). En consecuencia, las personas contratadas directamente por receptores de pagos directos no estarán sujetas a regulación ni evaluación. ${ }^{17}$

A continuación, exponemos los principales retos identificados por la Audit Commis- sion (2010) para los gobiernos locales ante la implementación y desarrollo del sistema SDS:

1. Los gobiernos locales han de ser realistas al estimar los costes y beneficios asociados a los presupuestos personales y sus implicaciones sobre la planificación:

- El Sistema de Apoyo Auto-dirigido puede generar ahorro únicamente en casos en los que los gobiernos locales estén soportando costes excesivos en los Servicios Sociales contratados, como consecuencia de una práctica de commissioning deficiente.

- La introducción de presupuestos personales pone en riesgo los contratos en bloque con entidades prestadoras de servicios sociales, al ampliarse la libertad y las posibilidades de elección de los usuarios.

- La prestación directa de servicios sociales está en riesgo por los mismos motivos y necesita ser revisada.

2. El sistema de asignación de recursos ha de ser factible desde el punto de vista presupuestario, transparente y equitativo para todos los grupos de usuarios de Servicios Sociales. Ha de proporcionar una cantidad indicativa en lugar de definitiva.

3. La introducción de los presupuestos personales supone un nuevo planteamiento para los departamentos de Servicios Sociales en cuanto a sus roles y relaciones:

- Tendrán que trabajar con las entidades prestadoras y otros grupos de interés para influir sobre los servicios ofrecidos y asegurar que éstos cubren las necesidades de los usuarios de presupuestos personales.

- Habrán de potenciar las funciones de información y asesoramiento para los receptores de presupuestos personales, incluso para aquellos que hacen frente al coste de sus servicios por sí mismos. Estas funciones pueden ser prestadas de forma directa o indirecta.

\footnotetext{
${ }^{17}$ No obstante, recientemente ha entrado en vigor la normativa Vetting and Barring Scheme, que se aplicará a aquéllos que trabajen con adultos y niños a partir de octubre de 2009. La principal novedad respecto a regulaciones anteriores, es que todos los agentes implicados (empleadores, gobiernos locales, reguladores profesionales y cualquier otro organismo) tienen la obligación de informar a la Autoridad de Salvaguarda de la Independencia (Independent Safeguarding Authority) cuando consideren que alguien ha causado daños o ha supuesto un riesgo para niños o adultos vulnerables. El Vetting and Barring Scheme es de aplicación en Inglaterra, Gales e Irlanda del Norte, mientras que en Escocia regirán otros acuerdos bajo el Protection of Vulnerable Groups (Scotland) Act 2007.
} 
- Deberán tomar medidas para combatir el fraude en el uso de los presupuestos personales sin desincentivar la innovación y el espíritu de la personalización que inspira al Sistema de Apoyo Auto-dirigido.

4. Los departamentos de Servicios Sociales han de asegurarse de que sus sistemas de información son adecuados para el desarrollo del Self-directed Support y no generan obstáculos burocráticos:

- Los planes financieros a medio plazo han de recoger las estimaciones de costes y demanda relativos a los presupuestos personales y las implicaciones de éstos sobre los presupuestos futuros.

- La emisión de información financiera y de gestión y el enfoque de control financiero deben adaptarse a los nuevos requerimientos.

- La revisión de los sistemas de información interna ha de asegurar su capacidad para ofrecer información relevante para la toma de decisiones y el control de la actuación.

En relación a este último aspecto, las asociaciones Local Government Improvement and Development (IDeA) ${ }^{18}$, Association of Directors of Adult Social Care Services (ADASS) y Local Government Association (LGA) ${ }^{19}$ (2010), han señalado que el diseño de mecanismos sólidos para entender y medir el éxito de los resultados alcanzados por el Sistema Self-directed Support constituye uno de los principales retos para los gobiernos locales en la actualidad.

\section{Conclusiones}

Nunca antes ha sido tan evidente la necesidad de introducir mejoras en la prestación de los Servicios Sociales públicos que hagan hincapié en la reducción de los costes y, en consecuencia, en la mejora de la eficiencia. La utilización de técnicas de comparación de buenas prácticas - benchmarking - que permitan trasladar formas de actuación, desde países pioneros como Reino Unido, puede conllevar una mayor cobertura de las necesidades socia- les acompañada de una mayor calidad y eficiencia. Por ello, se han descrito dos de dichas técnicas cuyo impacto en la prestación de Servicios Sociales en dicho país ha provocado importantes efectos en este sentido. Estas técnicas se centran en las políticas de prevención y en la elaboración de presupuestos personales para dar cobertura a la prestación social.

La implementación de las políticas de prevención en la prestación de los Servicios Sociales, provoca un gran efecto en la calidad de vida de los ciudadanos, si bien podría verse atenuado dada la inversión inicial que su puesta en marcha requiere. Puede decirse, por lo tanto, que favorece la reducción de costes y la generación de eficiencia en el medio y largo plazo.

Con respecto al diseño de los presupuestos personales, supone una revolución tanto en la prestación como en la financiación de los Servicios Sociales. Para su puesta en marcha se precisa un sistema de información que permita el cálculo del coste de prestación de dichos servicios, a partir del cual sería fijada la cantidad contemplada en dicho presupuesto personal. En relación al mismo, la contabilidad interna de estos departamentos debe ser lo suficientemente desarrollada como para permitir el cálculo y control de costes de las diferentes actividades, centros y del propio servicio prestado. Para garantizar la utilidad del sistema, tendría que ser lo suficientemente flexible como para evitar estrangulamientos derivados de un exceso de rigidez en la prestación y de una alta burocracia. Los planes financieros a largo plazo deben contemplar dicha información que, sin duda, condicionaría la toma de decisiones y la supervisión de la prestación, independientemente de cómo haya decidido el usuario gestionar el presupuesto atribuido, ya sea mediante pago directo, presupuesto personal gestionado por los departamentos de Servicios Sociales o bien mediante un presupuesto personal mixto.

${ }^{18}$ Local Government Improvement and Development (IDeA) es un organismo público, dependiente de la Local Government Association (LGA) en Inglaterra y Gales. Su finalidad es fomentar la mejora y la innovación del Gobierno Local, mediante el desarrollo conjunto de buenas prácticas a través del trabajo en red y el apoyo telemático.

${ }^{19}$ Local Government Association (LGA) representa a 423 entidades locales en el Reino Unido. Su objetivo es apoyar, promover y mejorar el gobierno local. 


\section{Referencias bibliográficas}

Antonnen, A. y Sipila, J. (1996). European social care services: is it posible to identify models?. Journal of European Social Policy, 6 (2), 87-100.

Association of Directors of Adult Social Services. (2009). Common Resource Allocation Framework (\& appendicies 1-3).

Audit Commission. (2010). Financial management of personal budgets: challenges and opportunities for councils. Summary of the national report. Londres.

Audit Commission. (2011). Improving value for money in adult social care. Londres.

Casado, D. y Fantova, F. (2007). Perfeccionamiento de los Servicios Sociales en España. Informe con ocasión de la Ley sobre autonomía y dependencia. Madrid: Colección de Estudios Fundación FOESSA y Cáritas Española Editores.

Department for Communities and Local Government. (2010). Local Government Financial Statistics, England, 2, Londres.

Department of Health. (2007). Putting People First. A shared vision and commitment to the transformation of Adult Social care. Londres.

Department of Health. (2009). Use of Resources in Adult Social Care: A guide for local authorities. Londres.

Department of Health. (2010). Local Authority Circular on the Personal Care at Home Act 2010 and Charging for Re-ablement. LAC (DH) (2010) 6, Londres.

Department of Health. (2010a). Prioritising need in the context of Putting People First: A whole system approach to eligibility for social care. Londres.

Department of Health. (2010b). A vision for adult social care: Capable communities and active citizens. Londres.

Department of Health. (2010c). Fairer Contributions Guidance: Calculating an Individual's Contribution to their Personal Budget. Londres.

García, I. (2007). La nueva gestión pública: evolución y tendencias. Presupuesto y Gasto público, 47, 38.

Glendinning, C., Jones, K., Baxter, K., Rabiee, P., et al. (2010). Home Care Re-ablement Services: Investigating the longer-term impacts (prospective longitudinal study). Kent: Social Policy Research Unit, University of Kent

Hampshire County Council. (2009). The Hampshire Model-transforming care support for aduts. A blueprint for change. Year One Plan. Winchester (United Kingdom).

Henwood, M. (19 de marzo, 2008). Individual budgets gathering steam. Community Care, Recuperado de: http://www.communitycare.co.uk.

Her Majesty Government. (2009). Shaping the Future of Care Together. Green paper.

Her Majesty Government. (2010). Building the National Care Service. Londres.

Hogwood, B. W. y Peters, B. G. (1985). The Patology of Public Policy. Oxford: Clarendon Press.

Hughes, O. E. (2003). Public Management Administration. An introduction. Nueva York: Macmillan Press Ltd.

Jennergren, L. P. (1981). Descentralization in organizations. En P.C.Nystrom y W.H. Starbuck (eds.): Handbook of Organizational Design. Vol. 1: Adapting Organizations to their Environments (pp. 39-59). Nueva York: The Free Press.

Kent, J., Payne, C., Stewart, M. y Unell, J. (2000). External Evaluation of the Home Care Re-ablement Pilot Project. Centre for Group Care and Community Care Studies, De Montfort University, Leicester.

Local Government Association. (2010). CAA evaluation: a sector perspective on year 1. Londres.

Local Government Improvement and Development, Association of Directors of Adult Social Care Services y Local Government Association. (2010): Personal budgets - checking the results. Londres.

Lombard, D. (Julio, 2008). Direct payment users not asking for references or CRB checks. Community Care. Recuperado de: http://www.communitycare.co.uk.

Mithran, S. (abril, 2009). Direct payments, personal budgets and individual budgets. Community Care. Recuperado de: http://www.communitycare.co.uk. 
Nottinghamshire County Council. (2010). Improvement Programme Report (2010). Nottingham (United Kingdom).

Nottinghamshire County Council. (2010a). Putting People First. Transforming social care in Nottinghamshire Newsletter Spring 2010.

Osborne, S. P. (2000). Public-Private Partnerships. Londres: Routledge.

Prowle, M. (2010). Managing and Reforming Modern Public Services. The Financial Management Dimension. Prentice Hall Financial Times. 